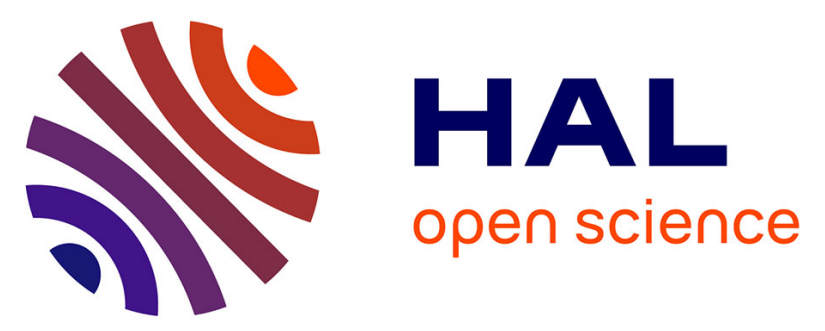

\title{
Amplification of the luminescence response in organic materials exposed to ionizing radiation
}

\author{
Maugan Michel, Licinio Rocha, Matthieu Hamel, Stéphane Normand
}

\section{To cite this version:}

Maugan Michel, Licinio Rocha, Matthieu Hamel, Stéphane Normand. Amplification of the luminescence response in organic materials exposed to ionizing radiation: A new method for ionizing radiation detection. 2012 IEEE Nuclear Science Symposium and Medical Imaging Conference (2012 NSS/MIC), Tom K. Lewellen, University of Washington Medical Center, Oct 2012, Anaheim, United States. pp.1753-1755, 10.1109/NSSMIC.2012.6551410 . cea-02509558

\section{HAL Id: cea-02509558 https://hal-cea.archives-ouvertes.fr/cea-02509558}

Submitted on 16 Mar 2020

HAL is a multi-disciplinary open access archive for the deposit and dissemination of scientific research documents, whether they are published or not. The documents may come from teaching and research institutions in France or abroad, or from public or private research centers.
L'archive ouverte pluridisciplinaire HAL, est destinée au dépôt et à la diffusion de documents scientifiques de niveau recherche, publiés ou non, émanant des établissements d'enseignement et de recherche français ou étrangers, des laboratoires publics ou privés. 


\title{
Amplification of the Luminescence Response in Organic Materials Exposed to Ionizing Radiation - A New Method For Ionizing Radiation Detection
}

\author{
Maugan Michel, Licinio Rocha, Matthieu Hamel and Stéphane Normand.
}

\begin{abstract}
Polymer-based scintillators present interesting features for the field of ionizing radiation detection, related to the high sensitivity of fluorescence techniques coupled to their manufacturing advantages. Organic materials can indeed be manufactured into large sensing areas with different geometrical conformations through low-cost fabrication techniques.

Widely used for sensing applications because of its high sensitivity, fluorescence has yet been further improved using technologies yielded by research in photonics. It has already been shown that the use of nanostructuration for sensing applications enables previously unattained precision.

Here we propose a new technique based on the manipulation of light using nanostructuration of the detection medium in order to enable the amplification of the sensitive material emission. This amplification of the scintillation signal is aimed at reducing the detection limit of low-energy beta emitters such as tritium, well-known issue of major importance.

The first step of our study, presented here, consists in demonstrating the ability of well known scintillators to emit in laser regime when optically excited in a Distributed Feedback scheme. They are, to our knowledge, the first of their kind.

The technique here presented, being usable, whatever the sample maximum emission wavelength, should also enable a simplification of the devices based on scintillators.
\end{abstract}

Index Terms-Ionizing radiation, Detection, Efficiency.

\section{INTRODUCTION}

$\mathbf{C}$ ONTROLLING light in dielectric materials is an important field of photonics. Widely studied for communication technologies, nano-modification of the material properties have found applications in fields ranging from lab-on-chip devices [1] to Martian exobiology [2].

Inducing a periodic variation in a dielectric material's properties in the optical wavelength scale can modify the material's behaviour towards light. Thus inducing gain, refractive index, or topology gratings can change the generation, absorption and propagation of light in the modulated medium. Following diffraction of light propagating in periodic media, privileged light channels can for example be defined. Combined with a primary light amplification phenomenon, in specific structures, great amplification can occur. Interaction of light with

Corresponding author. Tel: +33 1690825 27, E-mail address: maugan.michel@cea.fr (M. Michel)

Manuscript received November 15, 2012.

M. Michel is with CEA, LIST, Laboratoire Capteurs et Architectures Électroniques, F-91191 Gif-sur-Yvette, France. Telephone: 331690825 27, e-mail: maugan.michel@cea.fr).

L. Rocha, M. Hamel and S. Normand are with the CEA, LIST, Laboratoire Capteurs et Architectures Électroniques, F-91191 Gif-sur-Yvette, France. Email: licinio.rocha@cea.fr, stephane.normand@cea.fr nanostructured media can thus be implemented to design devices that are highly sensitive to any modification of the environment, with strong potential for detecting devices [3], [4].

\section{Controlling Light Through Nanoscale} STRUCTURATION - LASER EMISSION IN SCINTILLATORS

Population inversion, required so as to emit stimulated radiation [5] can be produced in conjugated molecules, through their $\pi$-delocalized electrons. Scintillators, containing such molecules could thus be used as lasing medium.

Appart from the cost reduction due to the use of organic compounds, these materials come with various permanent nanostructuration techniques, also cost effective. The device used for this study, seen in Fig. 2, generates transient gratings enabling real-time control of the periodic structures characteristics.

The diffraction of light in particular gratings can produce Distributed Feedback (DFB) laser emission in which the diffracted beam contra-propagates in the grating, exchanging energy with the propagating wave [6] all along the grating. Provided sufficient gain and excitation, the ensuing resonant structure provides an amplification in the material resulting in a laser emission (see Fig. 1). The emitted wavelength $\lambda_{\mathrm{em}}$ for this lasing condition to be verified, called the Bragg condition, is calculated using Eq. (1):

$$
\lambda_{\mathrm{em}}=\frac{2 n_{\mathrm{eff}} \Lambda}{p}
$$

Where $n_{\text {eff }}$ the effective refractive index of the medium where the grating exists and $p$ the diffraction order.

One method to achieve a refractive index grating is by using the Kerr effect. It is a non-linear optical effect taking place when high-amplitude polarization variation occurs in a dielectric medium. Through Eq. (2), it appears that through a change of light intensity, one can create a spatially modulated refractive index $\Delta n$, given by Eq. (2):

$$
\Delta n=n_{\mathrm{e}}-n_{\mathrm{o}}=\lambda K E^{2}
$$

Where $n_{\mathrm{e}}, n_{\mathrm{o}}$ and $K$ being the extraordinary and ordinary refractive index and the Kerr constant, respectively. A transient refractive index grating can easily be achieved by making two coherent beams of wavelength $\lambda_{\text {exc }}$ interfere at an angle $\theta$ in a material. The experimental setup for such transient grating is 

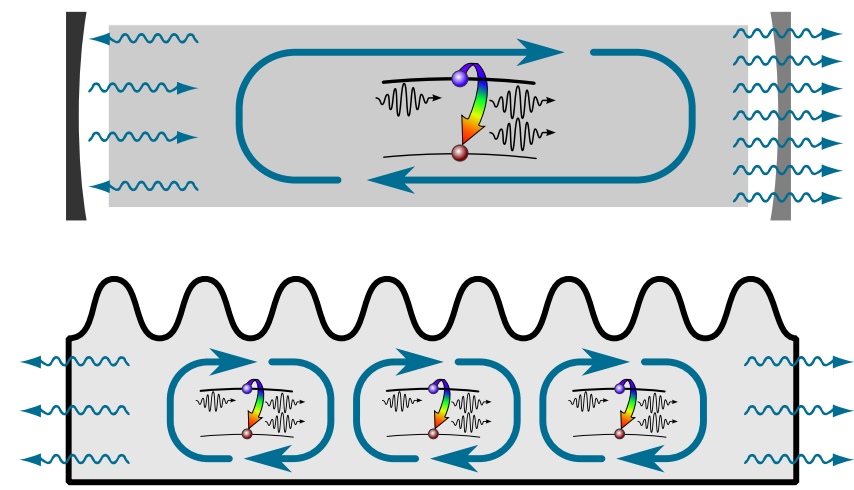

Fig. 1. Conventional (left) and Distributed Feedback (right) lasers' feedback schemes. While feedback in the gain medium (undergoing stimulated emission) in conventional lasers is provided by the mirrors, in a Distributed Feedback scheme, it occurs all along the grating.

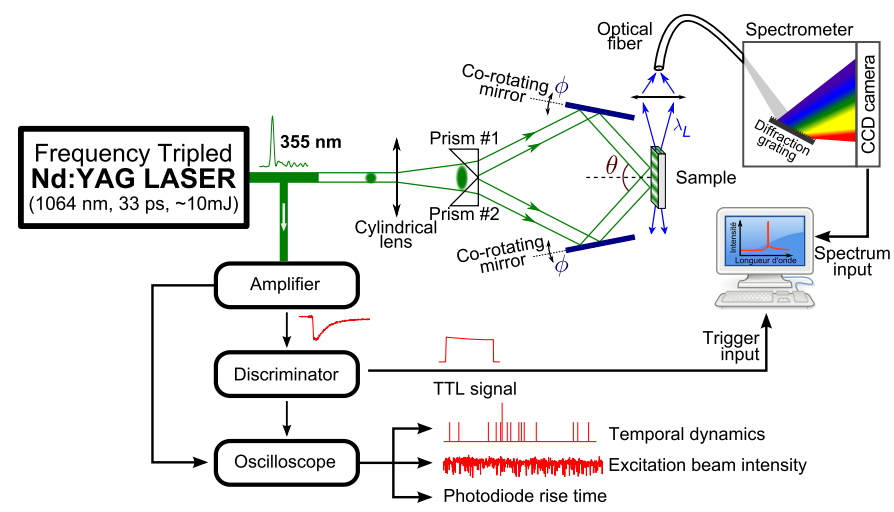

Fig. 2. Schematic of the dynamic Distributed Feedback grating experimental setup. The intereference pattern creates two gratings at the same time:

Prisms are UV-grade silica. Corotation angle of the mirrors is controlled through a linear translation stage.

Materials are excited with the second $(532 \mathrm{~nm})$ or third $(355 \mathrm{~nm})$ harmonics of a picosecond $\mathrm{Nd}^{3+}:$ YAG laser.
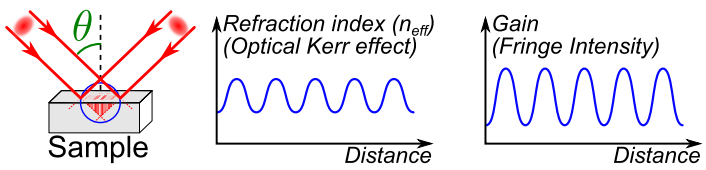

Fig. 3. Index and gain gratings created by the interference pattern of two coherent beams in a material.

presented in Fig. 2. The period $\Lambda$ of the grating is calculated using Eq. (3):

$$
\Lambda=\frac{\lambda_{\text {exc }}}{2 \sin \theta}
$$

As well as a refractive index grating, a gain grating is also created thanks to the periodic illuminance (see Fig. 3)

It appears from Eq. (3) that the emission wavelength is directly related to the beams intersection angle. Thus, changing the angle of the corotating mirrors enables continuous tuning of the emitted photon wavelength. Since there is a feedback between polarization of the medium and the propagating electromagnetic wave, it can be said that light can interact with light and thus control it[7].

Fig. 4 shows the molecular structure of the widely used Rhodamine 6G (Rh6G) laser dye, as well as its emission

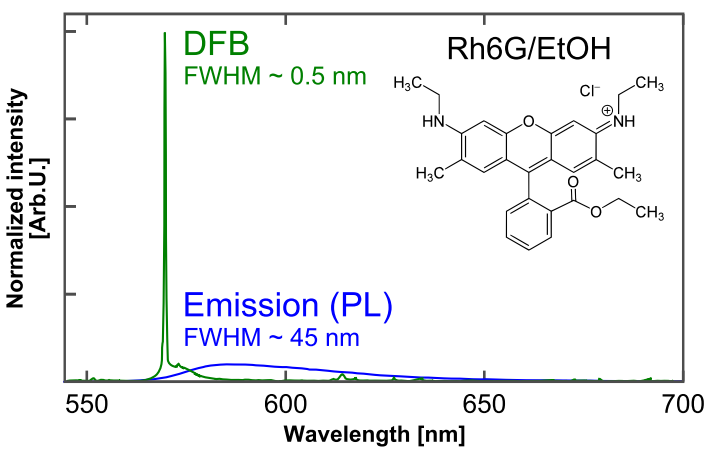

Fig. 4. Fluorescence and Distributed Feedback spectra and structure of Rhodamine $6 \mathrm{G}$ in Ethanol $\left(5 \cdot 10^{-3} \mathrm{M}\right)$. Fluorescence spectrum was measured using a Fluoromax 4 spectrofluorometer (HORIBA), $\lambda_{\text {exc }}=357 \mathrm{~nm}$. DFB excitation beam: $10 \mathrm{~mJ}$ doubled $(532 \mathrm{~nm}) \mathrm{Nd}^{3+}$ :YAG laser.

(photoluminescence) and a typical emission spectrum once the DFB conditions are verified. Along with the laser peak typically being one to two orders of magnitude more intense than the photoluminescence maximum, independently from the excitation beam intensity. Its full-width at half-maximum is approximately $0.5 \mathrm{~nm}$.

\section{Distributed FeEdback LASING OF COMMERCIAL LIQUID SCINTILLATORS}

As seen in Fig. 2, using a $10 \mathrm{~mJ}$ pulsed $\mathrm{Nd}^{3+}$ :YAG laser $(1064 \mathrm{~nm})$, an interference pattern is created in the sample, simultaneously generating gain and refractive index gratings.

The samples that are to be studied are Ultima Gold ${ }^{\mathrm{TM}} \mathrm{AB}$ (PerkinElmer, UGAB) and NE-213/BC-501A (Saint-Gobain Crystals), both widely used commercial liquid scintillators. Considering their absorption spectra, their excitation is obtained by frequency-tripling the $\mathrm{Nd}^{3+}$ :YAG laser $(\lambda=$ $355 \mathrm{~nm}$ ), allowing stimulated emission of these samples, thus lasing when the Bragg condition is verified.

As can be seen from Figs. 5 and 6, although not designed for this purpose, we report Distributed Feedback (DFB) lasing for both samples. The tuning of the DFB laser wavelength of Ultima Gold $^{\mathrm{MM}} \mathrm{AB}$ ranges over at least $40 \mathrm{~nm}$ (from $395.2 \mathrm{~nm}$ to $435.6 \mathrm{~nm}$ ). Also clearly appears the important amplification due to DFB when beam intersection is optimum. The ratio between DFB and stimulated emission peaks intensities, $\frac{\mathrm{DFB}}{\mathrm{SE}}$ is a good indicator of this amplification; values of 24 and 65 for Ultima Gold ${ }^{\mathrm{TM}} \mathrm{AB}$ and BC-501A respectively have been reached.

\section{CONCLUSION}

The results presented here were obtained using commercial liquid scintillators, which are not initially designed for laser applications. Laser emission has been demonstrated in scintillators using transient gratings. Other nanostructuration techniques are already being investigated.

The results of our preliminary investigation show promising features for the future of scintillating detectors. The results regarding the effect of ionizing radiation on distributed-feedback lasing will soon be published. 


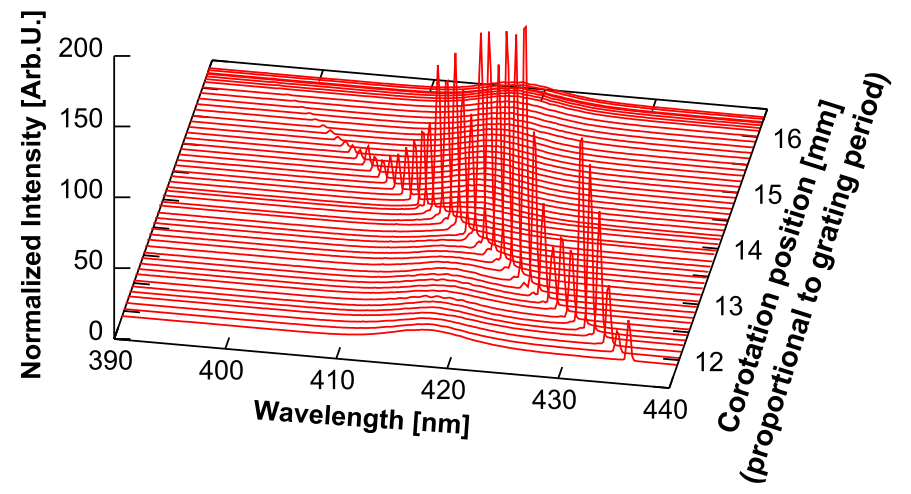

Fig. 5. Variation of the DFB peak wavelength vs. corotation linear stage position for Ultima Gold ${ }^{\mathrm{TM}} \mathrm{AB}$. The interfering angle is directly linked to the the position of the linear translation stage (coupled to both mirror's axes). The decrease in peak intensity circa $428 \mathrm{~nm}$ is due to non-optimized structuration. DFB excitation beam: $10 \mathrm{~mJ}$ tripled $(355 \mathrm{~nm}) \mathrm{Nd}^{3+}$ :YAG laser.

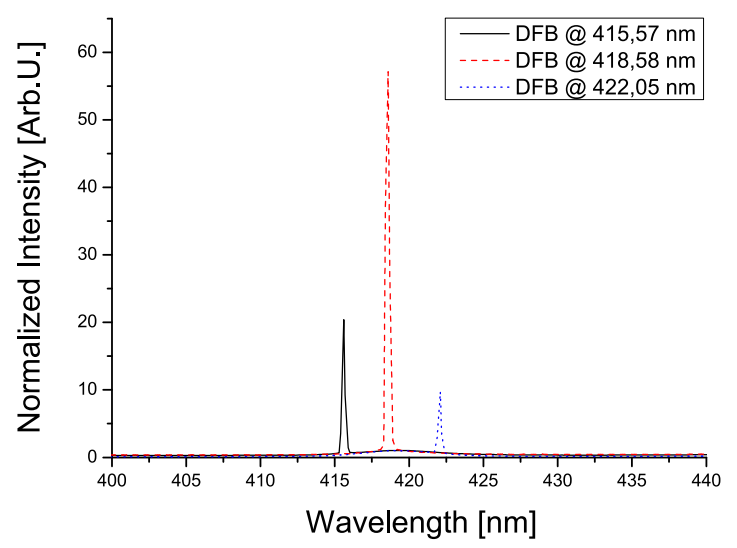

Fig. 6. Variation of the DFB peak wavelength $v s$. corotation linear stage position for BC-501A. DFB excitation beam: $10 \mathrm{~mJ}$ tripled $(355 \mathrm{~nm})$ $\mathrm{Nd}^{3+}:$ YAG laser.

\section{REFERENCES}

[1] M. Gersborg-Hansen and A. Kristensen, "Optofluidic third order distributed feedback dye laser," Applied Physics Letters, vol. 89, no. 10, p. 103518, 2006. [Online]. Available: http://link.aip.org/link/?APL/89/103518/1

[2] P. Coll, M. Cabane, P. Mahaffy, and W. Brinckerhoff, "Sample analysis at Mars," in 35th COSPAR Scientific Assembly, vol. 35, 2004, p. 3605. [Online]. Available: http://msl-scicorner.jpl.nasa.gov/Instruments/SAM/

[3] W. Zeller, L. Naehle, P. Fuchs, F. Gerschuetz, L. Hildebrandt, and J. Koeth, "DFB Lasers Between $760 \mathrm{~nm}$ and 16 micrometer for Sensing Applications," Sensors, vol. 10, no. 4, pp. 2492-2510, 2010. [Online]. Available: http://www.mdpi.com/1424-8220/10/4/2492/

[4] A. Rose, Z. Zhu, C. F. Madigan, T. M. Swager, and V. Bulovic, "Sensitivity gains in chemosensing by lasing action in organic polymers," Nature, vol. 434, no. 7035, pp. 876-879, Apr 2005, attenuated lasing in optically pumped SOP thin films displays a sensitivity to vapours of explosives more than 30 times higher than is observed from spontaneous emission.

[5] A. Einstein, "Zur Quantentheorie der Strahlung," Physikalische Zeitschrift, vol. 18, pp. 121-128, 1917.

[6] H. Kogelnik and C. V. Shank, "Stimulated emission in a periodic structure," Applied Physics Letters, vol. 18, no. 4, pp. 152-155, February 1971.

[7] G. Boulon, "Génération dimpulsions lasers ultracourtes jusquà la femtoseconde," Techniques de l'ingénieur., vol. AF3282, no. AF3282, pp. 1-24, 2006. [Online]. Available: http://www.techniquesingenieur.fr/base-documentaire/sciences-fondamentales-th8/physique- chimie-ti053/generation-d-impulsions-lasers-ultracourtes-jusqu-a-lafemtoseconde-af3282/ 